\title{
Mental Workload Analysis Associated with Emotional Design in E-learning Contexts: Combining EEG and Eye-tracking Data
}

\author{
Chen-Nan $\mathrm{Wu}^{1}$, Yang $\mathrm{Liu}^{1,}{ }^{*}$, Xiang Guo ${ }^{1}$, Wei-Feng $\mathrm{Ma}^{1}$ and Tian-Shui Zhu ${ }^{1}$ \\ ${ }^{1}$ Zhejiang University of Science and Technology, Hangzhou, 310000, China \\ *Corresponding Author: Yang Liu. Email: liuyang@zust.edu.cn
}

KEYWORDS

Mental Workload;

EEG;

Eye-tracking;

Machine Learning;

Multimodal

\begin{abstract}
In this study, we designed an online course mental workload induction experiment, which recorded EEG and Eye-tracking data of participants synchronously, aiming to investigate the association between different online course design principles and multimodal physiological features, and apply machine learning technology to classify mental workload states induced by different design principles. This paper systematically reviews three kinds of EEG and Eye-tracking features used for mental workload classification, compares the accuracy of mental workload classification between single-modal and multimodal features, modifies the mental workload index proposed by Pope et al. to monitor the variation of mental workload in E-learning contexts, and reduces the dimensions of features for more convenient use in daily life. From the results of the experiment, it's demonstrated that (1) The classification ability of wavelet power features and entropy features are better than that of Eye-tracking in E-learning Contexts. (2) Multimodal physiological data can significantly improve the accuracy of mental workload classification in E-learning contexts, and (3) correlation-based feature selection (CFS) was employed to rank all features in descending order, when the feature dimension is reduced to 30, the optimal average classification accuracy obtained by linear-SVM can reach $88.1 \%$, which greatly reduces the dimensions of feature while maintains a high accuracy. Furthermore, the EEG frequency bands that are highly correlated with mental workload are analyzed and the correlation between different brain areas and mental workload is discussed. All these results lay the foundation for continuous monitoring of participants' mental workload and make it possible to endow computers with the ability of mental workload understanding in E-learning contexts, which will remarkably enhance the learning efficiency and performance of participants during the pandemic.
\end{abstract}

\section{Introduction}

The recent global spread of COVID-19 has had a significant impact on both the educational and professional milieux around the world. As students are forced to study from home, a growing number of Elearning resources have emerged, with varying degrees of effectiveness and quality. Online courses are a key element of E-learning. According to the classification of "cognitive load" by Sweller et al.[1-5], total cognitive load is the sum of three cognitive loads, respectively: Intrinsic Cognitive Load (ICL), Extraneous Cognitive Load (ECL) and Germane Cognitive Load (GCL), as illustrated in Figure 1.

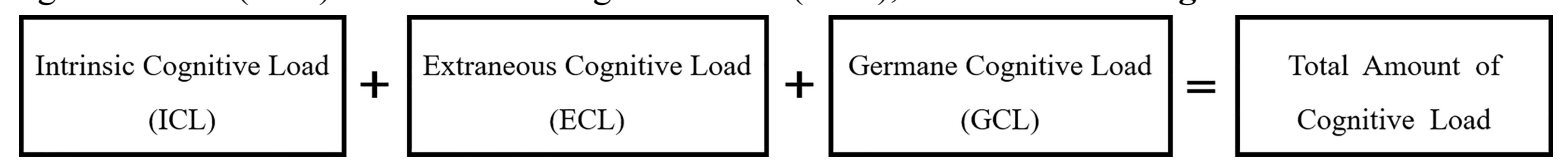

Figure1: Horizontal classification of cognitive load

Extraneous Cognitive Load (ECL), also known as ineffective cognitive load, is believed to be caused by psychological activities that do not contribute directly to learning and is mainly attributed to the manner with which learning materials are arranged and presented. This additional cognitive load occurs when learning materials are organized or presented in a way that does not directly relate to, and therefore 
interferes with, schema-building or automation. Because the cognitive resources required by ECL occupyor even overload - the learner's working memory capacity, ECL may hinder learning and thus affect the learning effect.

According to Cognitive Load Theory, people have limited cognitive resources, and any learning and problem-solving activity consumes these resources, which in turn creates a cognitive load. Therefore, the main purpose in the research of Cognitive Load Theory is to control the working memory load in the teaching process, in order to minimize the Intrinsic Cognitive Load and Extraneous Cognitive Load that hinders learning and maximize the Germane Cognitive Load that promotes learning, thus enabling participants to optimize their limited cognitive resources to achieve the best possible learning effect.

Cognitive load is measured by three dimensions: mental load, mental effort, and performance, in which mental workload shares the cognitive load induced by tasks and contexts. In recent years, researchers have been increasingly interested in maintaining an optimal mental workload in the performance of cognitive tasks[6][9]. Many laboratories have conducted research on the monitoring of mental workload under multi-task contexts[7], in order to maintain participants' mental workload at an optimal level. According to the Cognitive Load Theory, the differences in mental workload induced by task complexity are caused by Intrinsic Cognitive Load (ICL), yet ECL - that is, extraneous mental load created by the manner in which online courses are designed - can also influence the participants' total mental-workload "burden." This dynamic has not always been taken into account in the body of the previous research.

There have been extensive studies on the effects of mental workload on human behavior and physiology. Changes in participants' mental workload can be correlated with observed in their behavior, such as a decline in academic performance, or inability to focus. Participants' psychological and physiological parameters can also be monitored for changes, such as increases or decreases in oscillations at different EEG frequency bands caused by variances in mental workload. EEG activity in the theta (4-8 $\mathrm{Hz}$ ) and alpha $(8-11 \mathrm{~Hz})$ range seem to involve higher brain functions, including working memory, executive control, and concentration[8-10]. The collection of EEG data has been proven to be an effective, non-invasive method for monitoring memory load and is highly sensitive to different cognitive states[1113]. Eye-tracking is also a commonly-used standard in analyzing the effectiveness of teaching materials. Previous studies have confirmed that pupil dilation is a sensitive indicator of variations in mental workload[14-19]. It is believed that an analysis integrating eye-tracking and EEG data can provide further insight.

So far, many studies have proposed indices of mental workload, yet these are not based on E-learning contexts. For example, Pope et al. used (beta power)/(alpha power + theta power) to measure the task participation of operators in automated systems[20-21], and Anu Holm et al. used (theta Fz)/(alpha Pz) to predict cognitive changes in the brain during multi-tasking[22]. Moreover, classical studies of mental workload often require an abundance of electrodes to monitor EEG activities, while in the context of practical applications, it is necessary to seek a simple, less-intrusive and easy-to-operate method to reduce the interference of EEG monitoring equipment on the participants' learning process - and, by extension, the attendant results. In addition, many scholars have attempted to integrate multimodal physiological data to their studies in order to improve the recognition accuracy of human search intention, action intention or various cognitive state. Further studies are required in order to improve the performance in the assessment of mental workload to promote E-learning, as impacted by a variety of online course design principles.

In consideration of the above issues, this study is supported by Cognitive Load Theory and explores the association between different online course design principles and participants' multimodal features based on both EEG and eye-tracking data in the learning process. The study aims to use machine learning technology to classify cognitive states induced by different design principles.

The online course design principle is based on the emotional design theory of multimedia learning[2329]; the experiment employs online course design principles that feature either positive emotional design or neutral emotional design. According to previous studies[30-35], applying the principles of emotional design to online courses can induce positive emotions, and positive emotions can in turn facilitate learning. 
During the experiment, we simultaneously recorded participants' EEG and eye-tracking data. Nonlinear dynamics and wavelet transform were used to analyze the EEG data, and the relevant eyetracking measures were extracted in order to obtain the feature that can most effectively evaluate the participants' mental workload state. Machine learning techniques were used to identify "high" and "low" mental workload states induced by online resources using one of two emotional design principles (either "positive" or "neutral"). Before the experiment began, all participants took part in a learning simulation of the online course using a variety of different emotional designs and wearing an EEG device in front of a screen eye tracker. At the same time, we collected data on participants' in-class performance (test scores) as well as subjective, self-reported evaluations of the perceived difficulty of the course.

\section{Material}

\subsection{Participants}

42 undergraduate students from Zhejiang University of Science and Technology participated in the study, which was approved by the school and advisors; informed consent was secured from all participants. All participants had studied $\mathrm{C} / \mathrm{C}++$, but none had studied Python. The participants were randomly divided into two groups, according to $\mathrm{C} / \mathrm{C}++$ grades they got in their $\mathrm{C} / \mathrm{C}++$ programming course. It can be assumed that the participants in the two groups had the same prior knowledge of Python. Participants with (1) a history of major craniocerebral injury or neurological disease and (2) low arousal levels prior to the trial phase were excluded from the experiments[36].

\subsection{Experiments}

Online courses on the topic of Python were used as stimulus materials for the experiments. The design principles of the online courses (either "neutral" emotional design or "positive" emotional design) served as the independent variable, while the mental workload of the participants was the dependent variable. Before beginning, the staff presented the experiment in its entirety to the participants, and the participants in turn signed the informed consent. After cleaning the scalps of the participants, we let them put on their own electrode caps before we injected conductive adhesive to the electrodes and calibrated the eyemovement camera. We then asked participants to close their eyes for three minutes so we could take a baseline measurement. After these measurements were taken, the participants began the formal experiment: They were randomly assigned different online courses employing different emotional design principles (either neutral or positive). The entire process lasted about 10 minutes. Upon completion of the experiment, participants filled out the mental workload scale (NASA-TLX) and completed in-class tests[37-38]. Finally, we statistically analyzed the mental workload scale (NASA-TLX) and in-class tests, and found that the two emotional designs did induce distinct cognitive states as well as differences in learning performance (neutral emotional design, NASA-TLX $=5.20 \pm 1.54$, score $=3.73 \pm 1.76$; positive emotional design, NASA-TLX $=4.71 \pm 1.34$, score $=4.19 \pm 2.51$ ).

\section{Methods}

The combined recording of EEG and eye-tracking data obtained in the experiment were analyzed in a variety of ways, including signal preprocessing, feature extraction, feature dimension reduction, and mental workload classification. An overview of the detailed analysis procedure is presented in Figure 2. 


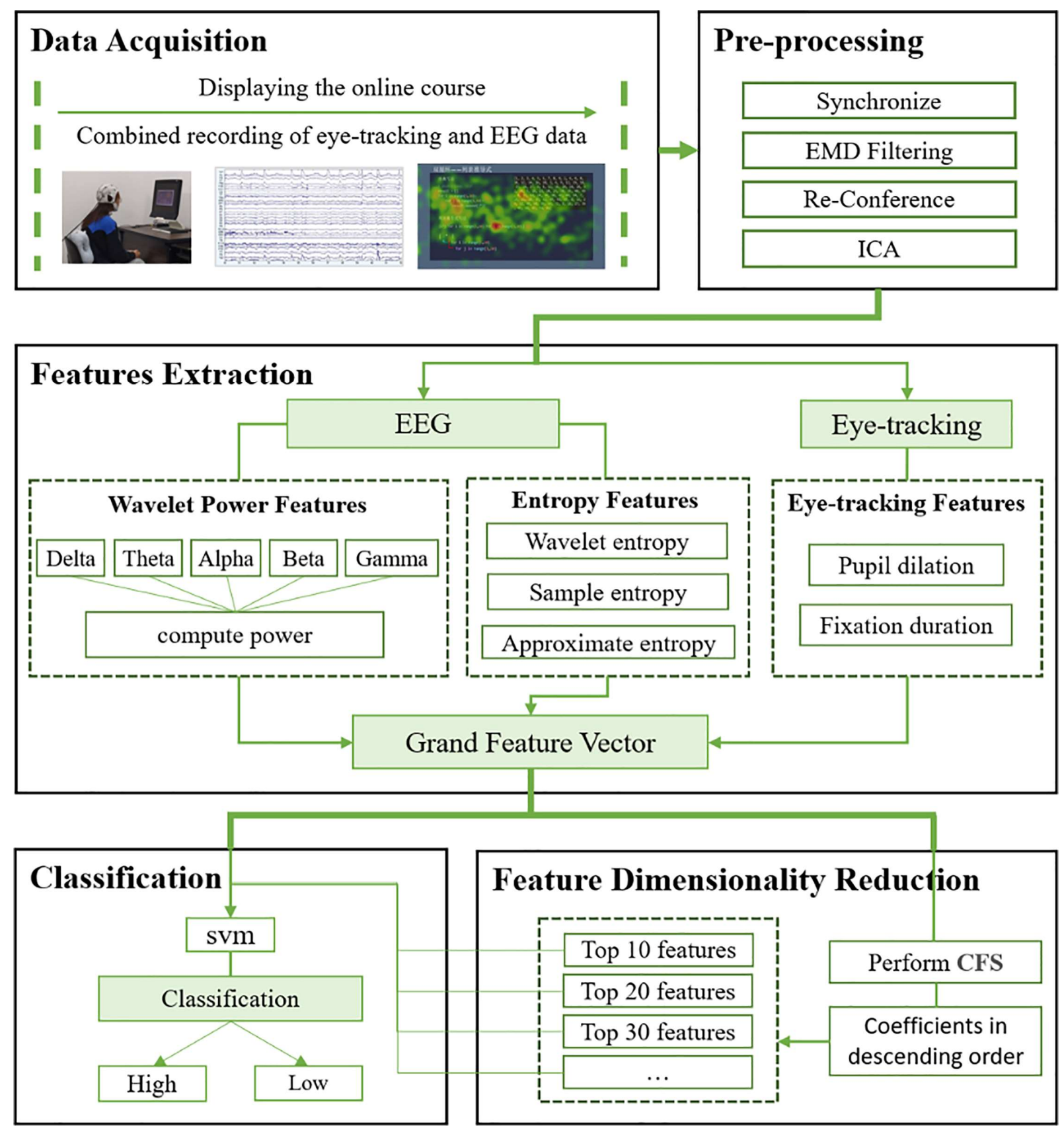

Figure 2: The procedure of data processing.

\subsection{Signal preprocessing}

The EEG collection device used in this study was OpenBCI and the eye-tracking equipment was Tobii T120. The original EEG and eye-tracking data were imported into MATLAB for synchronizing and preprocessing. The start and end markers of each stimulus in the EEG and eye-tracking data were used as synchronization events. The eye-tracking data was integrated into the EEG data as additional channels. For the EEG data, we used Empirical Mode Decomposition(EMD) to decompose and filter the signal, as EMD can be used to analyze the main components of the signal in an adaptive manner; then, the average reference was applied to the EEG data; finally, we conducted Independent Component Correlation Algorithm(ICA) 
to eliminate artifact components (e.g. blinking, muscle tension) [39]in order to ensure data hygiene. ICA is known to be suitable for removing artifacts in free-viewing contexts; ERP studies have demonstrated the efficiency of ICA, making it the most popular technique for removing ocular artifacts[40].

\subsection{Feature extraction}

\subsubsection{Nonlinear dynamics}

EEG data are highly complex and nonlinear. In recent years, many researchers have proposed methods for analyzing EEG using nonlinear dynamics theory. Among them, approximate entropy and sample entropy are important tools used to quantify the complexity of a time series. They can keep the information captured in an original time series as "whole" as possible, thus proving to be more effective than other methods in this respect[41-42].

\section{(a) Approximate entropy}

Approximate entropy, proposed by Pincus in 1991, can be used to consider the complexity and integrated degree of a sequence. It uses a non-negative number to represent the complexity and irregularity of a time series, and can reflect the occurrence rate of new sequence information in the time series. If the time series is more irregular and complex, the corresponding approximate entropy is larger [43-44].

A time series $\{x(n)\}=\{x(1), x(2), x(3), \ldots, x(N)\}$ consisting of $N$ pieces of data was defined, and the corresponding approximate entropy was calculated according to the following steps:

(1) Each $\mathrm{t}$ successive data in a time series can form a t-dimensional vector, that is:

$$
\left\{x_{t}(j)\right\}=\{x(j), x(j+1), \ldots, x(j+t-1)\}, 1 \leq j \leq n-m
$$

(2) The distance between sequences $\left\{X_{t}(j)\right\}$ and $\left\{X_{t}(k)\right\}$ is denoted as $d\left[X_{t}(j), X_{t}(k)\right]$; it is the largest absolute value of the difference of each corresponding position element of two time series vectors, that is:

$$
d\left[X_{t}(j), X_{t}(k)\right]=\max _{p=0,1, \ldots, t-1}\{|x(j+p)-x(k+p)|\}
$$

(3) Given a positive threshold w for time series $\left\{X_{t}(j)\right\}$ and $\left\{X_{t}(k)\right\}$, if the distance does not exceed the threshold $\mathrm{w}$, they are considered similar. Count the number of similarities between the time series $\left\{X_{t}(j)\right\}$ and other time series, and calculate its proportion to the total distances.

$$
\left.\emptyset^{\prime}{ }_{j}(w)=\frac{1}{N-t-1}\left\{\text { the number of } d\left[X_{t}(j), X_{t}(k)\right]\right\}<w, 1 \leq j \leq N-t+1, j \neq k\right\}
$$

(4) For each $\emptyset_{j}^{\prime}(w)$, calculate its logarithmic average:

$$
\emptyset^{\prime}(w)=\frac{1}{N-t-1} \sum_{j=1}^{N-t+1} \ln \left[\emptyset^{\prime}{ }_{j}(w)\right]
$$

(5) Increase to $t+1$ dimension, repeat Step $1-4, \emptyset^{t+1}(w)$ can be calculated as:

$$
\emptyset^{t+1}(w)=\frac{1}{N-t} \sum_{j=1}^{N-t} \ln \left[\phi_{j}^{t+1}(w)\right]
$$

(6) The approximate entropy is defined as:

$$
\operatorname{ApEn}(t, w)=\lim _{N \rightarrow \infty}\left[\emptyset^{\prime}(w)-\emptyset^{t+1}(w)\right]
$$


(b) Sample entropy

Compared to approximate entropy, sample entropy has two advantages: (1) The calculation of sample entropy does not depend on the duration of data; and (2) Sample entropy displays greater consistency. The smaller the sample entropy, the higher the sequence's self-similarity; the greater the sample entropy, the more complex the sample sequence[45]. Sample entropy was calculated as follows; all steps are the same as those for calculating the approximate entropy.

$$
\begin{gathered}
\emptyset^{t}(w)=\frac{1}{N-t+1} \sum_{j=1}^{N-t+1} \emptyset_{j}^{t+1}(w) \\
\operatorname{SampEn}(t, w)=\lim _{N \rightarrow \infty}\left[\emptyset^{\prime}(w)-\emptyset^{t+1}(w)\right]
\end{gathered}
$$

\subsubsection{Wavelet transform}

Studies have shown that the change in mental workload is closely related to the power spectral density of EEG signal channels[46]. Wavelet transform is a relatively new method for time-frequency analysis, which maintains the idea of Short-time Fourier Transform (STFT) local signal processing, and overcomes the shortcoming that the window size cannot be changed, making it an ideal tool for signal time-frequency analysis and processing. By using Daubechies wavelet as the basis function of wavelet transform, the EEG signal was decomposed into five sub-bands; in this manner, the frequency components of five frequency bands in the EEG signal could be extracted. For EEG signals from each channel, three features were extracted from the wavelet coefficients of each sub-band, respectively: wavelet energy, wavelet energy ratio (the ratio of the energy of each sub-band to the total combined energy of all sub-bands) and wavelet entropy, which are defined as:

(1) wavelet energy

$$
E(i)=\sum_{j=1}^{n_{i}} D_{i, j}^{2}
$$

(2) wavelet energy ratio

$$
R(i)=E(i) / \sum_{j=1}^{n} E(j)
$$

(3) wavelet entropy

$$
W_{e}=\sum_{i=1}^{n} R(i) \ln R(i)
$$

\subsection{Feature dimensionality reduction}

When using the machine learning model in data training, it is necessary to consider data size and data dimension. In the practical application scenario, large quantities of data and dimensions can cause machine learning models to run slowly and overload hardware devices. In addition, the issue of "dimension disaster" can arise when the dimensions are large. At present, there are two main types of dimension-reduction methods - the first being the methods represented by Principal Component Analysis (PCA), which damage the original data structure in order to extract the main features of the data, and the second being the correlation analysis of data, where certain laws are followed in the down-selection of weak correlation features. 
Existing studies have shown that unsupervised dimension reduction methods such as PCA are not always effective, while the feature selector based on correlation is a supervised method of dimension reduction, through which each feature will obtain a coefficient. We calculated the correlation coefficient between feature and label of each channel in each frequency band, in order to express its correlation with mental workload. A large value of correlation coefficient indicates a strong correlation of the feature with the target data, which can be used to select feature. By ranking these coefficients in descending order, the features most relevant to cognition can be identified. The correlation coefficient of each feature with cognition is calculated as follows:

$$
R_{r_{i} y}=\frac{C_{r_{i} y}}{\sqrt{C_{r_{i}} C_{y}}}
$$

In this index, $\mathrm{C}$ stands for "covariance" and $\mathrm{R}$ stands for "correlation coefficient."

\subsection{Classification}

The construction process of the mental workload identification model includes data acquisition, cognition-related feature extraction, feature dimension reduction, and classifier model construction. After the first three steps were completed, the final task was to design an effective cognitive classifier model according to some criteria of classification performance. In order to obtain accurate recognition and verify the effectiveness of the feature extraction and dimension reduction algorithm, the classifier is used to achieve the classification of predefined cognitive categories[47]. The accuracy of mental workload identification mainly depends on the effectiveness of the developed classifier.

Support Vector Machine (SVM) is a widely used classifier which has shown many unique advantages in solving nonlinear and high-dimensional pattern recognition, and can be widely used in other machinelearning problems. When SVM is used, a set of training samples needs to be given, where each sample contains several features and is accompanied by a property label clarifying to which of the two categories it belongs. SVM was realized in the following way - a data set with labels is given, with the task of finding a linear plane $w^{T} x_{i}+b$ meeting the following condition:

$$
y_{i}\left(w^{T} x_{i}+b\right) \geq 1, i=1,2, \ldots, N
$$

$x_{i}$ stands for feature vector and $y_{i}$ stands for its corresponding label.

\subsection{Statistical Analyses}

In order to determine whether the experimental results were caused by random errors, we conducted a significance test on the extracted features. We used an analysis of variance (ANOVA) model to compare the significance of features extracted from different emotional designs, and the results showed a significance level of $\mathrm{p} \leq 0.05$, which demonstrates that there are indeed significant differences in EEG and eye movement features contingent on the independent variable (online courses with different emotional designs). This confirms that these data can be used to distinguish the high and low loads induced by different emotional designs and reflect the accuracy of the machine-learning algorithm.

\section{Results}

\section{1 Feature extraction}

\subsubsection{Entropy feature}

In this paper, we studied two different nonlinear dynamic features of EEG signals (approximate entropy and sample entropy) and compared them with wavelet entropy. The average approximate entropy, sample entropy and wavelet entropy of all participants with different emotional designs in Figure 3 reveal 
that the approximate entropy clearly distinguishes the two kinds of emotional designs, while the distinction performance of sample entropy and wavelet entropy is less obvious.

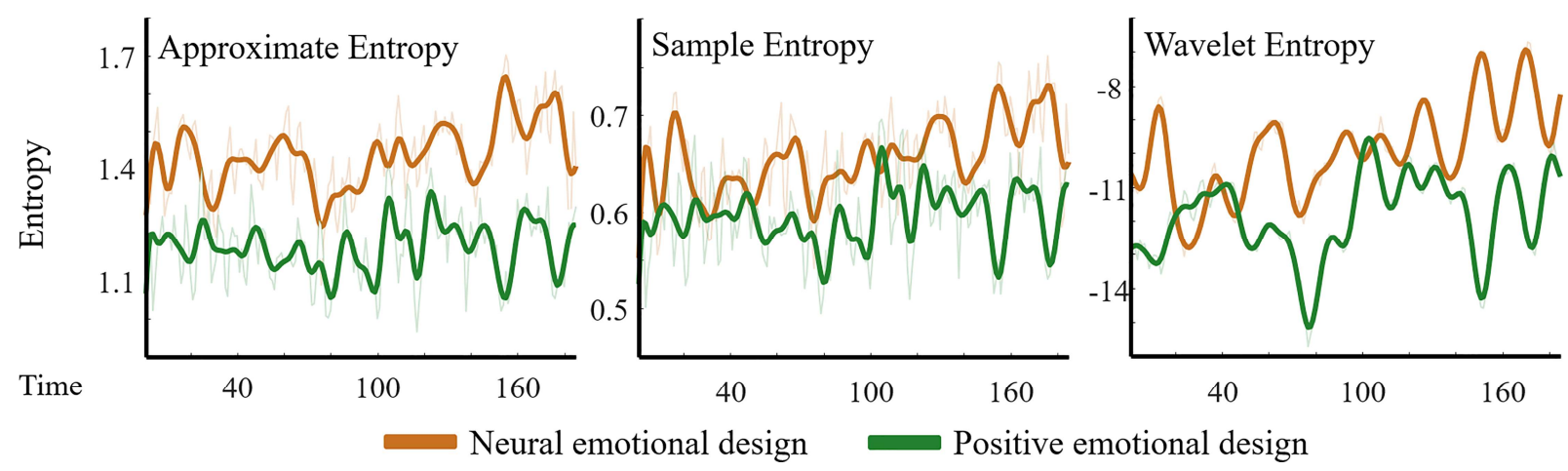

Figure 3: The average approximate entropy, sample entropy and wavelet entropy of all participants engaging with online courses of two different emotional designs

\subsubsection{Wavelet power feature}

After implementing wavelet transform on the EEG data and dividing it into five frequency sub-bands, we calculated average values of power for each frequency band and emotional design, as shown in Figure 4. It is apparent from this figure that the effects of emotional design on the frequency band power of theta, alpha, beta and gamma are significant; theta, alpha, beta and gamma frequency band power can clearly help to distinguish between the two types of emotional designs used in the online course. Of note, positive emotional design correlates to higher theta and alpha frequency band power and lower beta and gamma frequency band power - that is, theta and alpha frequency band power are positively related to positive emotional design and beta and gamma frequency band power are negatively related to positive emotional design.
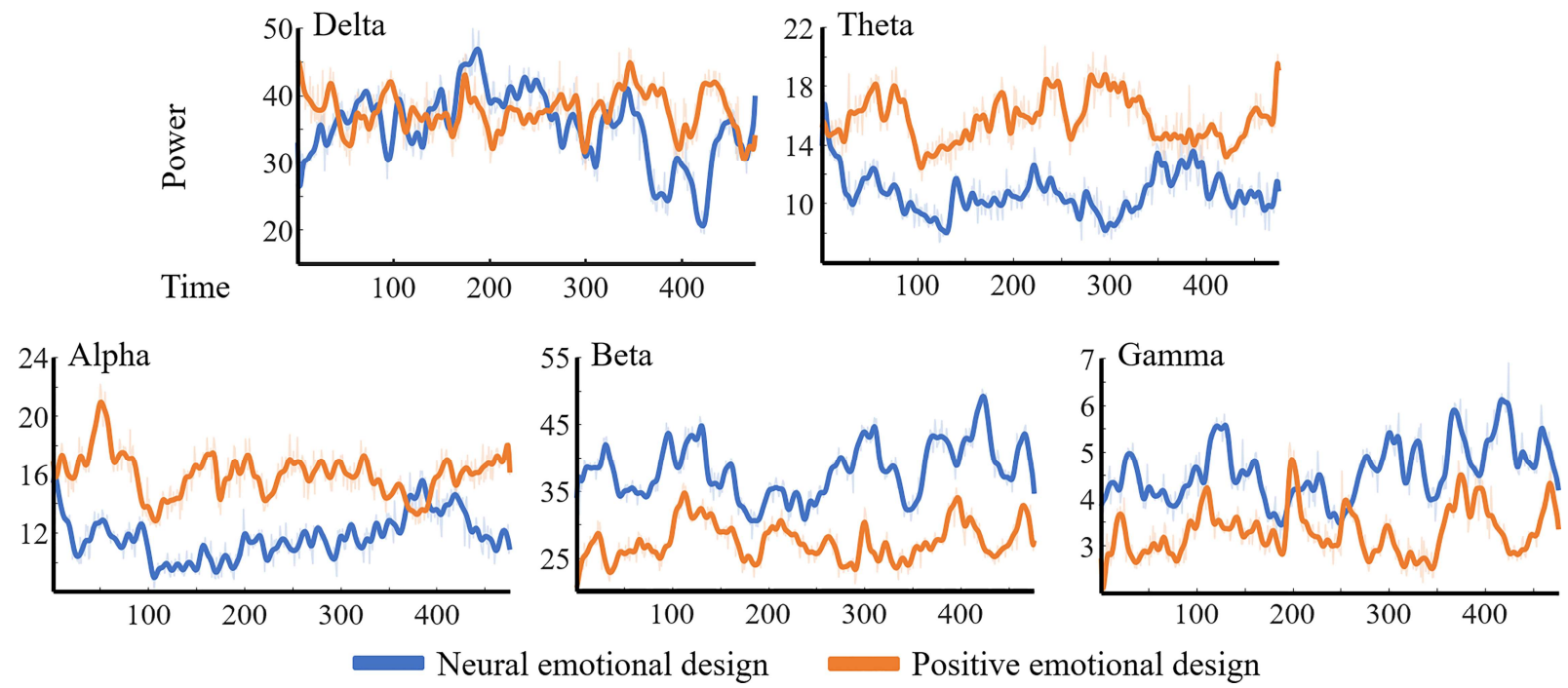

Figure 4: The average values of power for each frequency band and emotional design

\subsubsection{Eye-tracking feature}

Based on previous research[48], pupil dilation and fixation duration can be regarded as the key features of eye-tracking. Pupil dilation is closely related to human emotion and cognitive processing; for example, the higher the difficulty of text comprehension and the greater the memory load during reading, the greater the likelihood that the pupils will be enlarged. Fixation duration, on the other hand, is related to the degree 
of processing required to comprehend the material. Figure 5 exhibits the mean pupil dilation and fixation duration of all participants, with each colored bar representing the type of emotional design used.

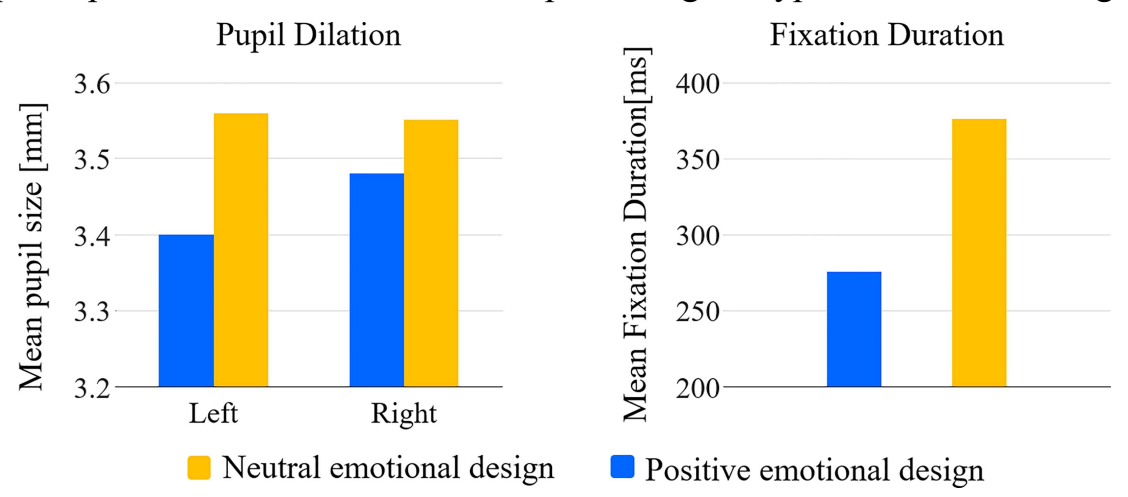

Figure 5: The average pupil dilation and duration of fixation of all participants while engaging with "neutral" and "positive" emotional designs

The data show that the average pupil dilation of participants assigned to the online course using positive emotional design is significantly smaller than that of participants assigned to the course using neutral emotional design -indicating that positive emotional design requires less cognitive processing when the content is held constant. With regard to fixation duration, the online course using positive emotional design correlates with a shorter average fixation duration compared to the course using neutral emotional design. These data indicate that learning material employing neutral emotional design consumes more of the participant's time and attention when compared to courses using positive emotional designwhich also suggests that online courses with positive emotional design are less likely to cause feelings of fatigue on the part of the learner.

\subsection{Result of mental workload classification}

\subsubsection{Result of mental workload classification based on single-modal features}

Linear-SVM classifiers were applied to classify three features and determine which was best at discriminating between different levels of mental workload induced by online courses employing either positive or neutral emotional design. When training the SVM model, the attribution of each sample under different classification criteria was used as an attribute label to compose the sample population. $50 \%$ of the sample were randomly selected for training, while the remaining $50 \%$ were used as test samples to evaluate classification accuracy.

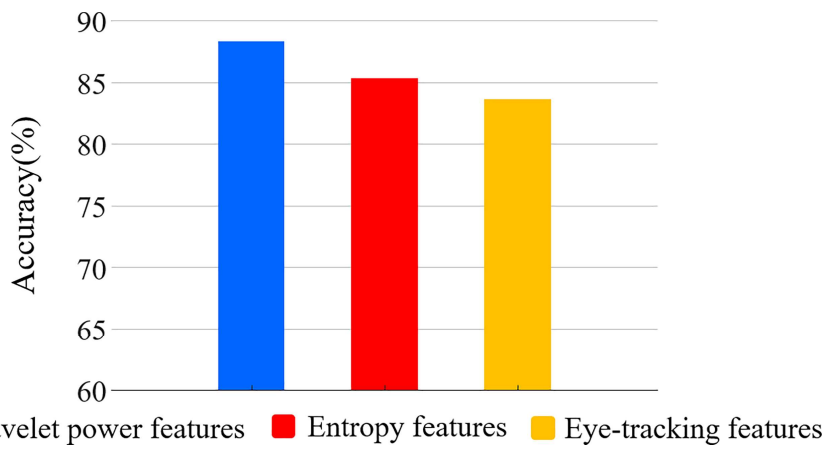

Figure 6: Comparison of classification accuracy of entropy feature, wavelet power feature and eye-tracking feature with linear-SVM 
Figure 6 exhibits the classification accuracy of the entropy feature, wavelet power feature and eyetracking feature. The results show that for each feature, accuracy was greatest when using the wavelet power feature, followed by the entropy feature and, lastly, the eye-tracking feature.

\subsubsection{Result of mental workload classification based on multimodal features}

The classification accuracy of a single-modal feature (either EEG or eye-tracking) and multimodal feature are, respectively, $88.6 \%$ (EEG), 83.6\% (eye-tracking), and 91.7\% (multimodal). Figure 7 illustrates the confusion matrix of the classification result. The classification performance based on EEG feature obtained by the SVM classifier is obviously better than eye-tracking feature. However, integrating EEG and eye-tracking to form a single multimodal feature boosts classification accuracy to $91.7 \%$ - translating to an increase of $3.1 \%$ compared to the single-modal feature based on EEG, and $8.1 \%$ compared to the single-modal feature based on eye-tracking. These data further confirm that the fusion of EEG and eye movement can enhance the capacity of the model to accurately classify mental workload.

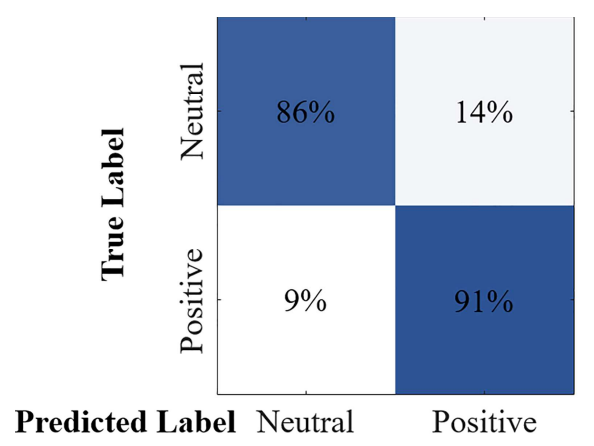

(a)

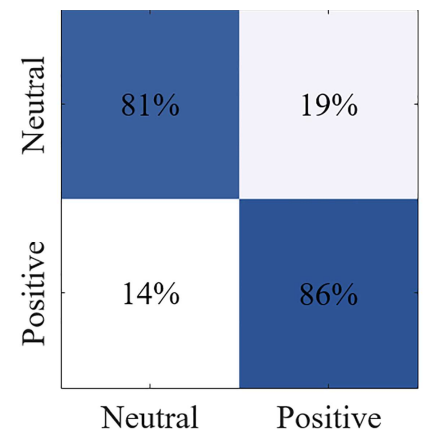

(b)

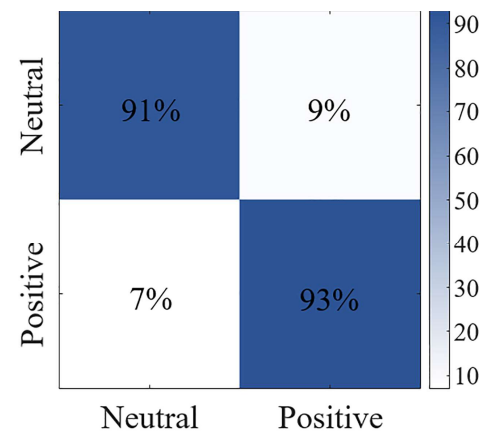

(c)

Figure 7: (a) Confusion matrix for single-modal feature (based on EEG) (b) Confusion matrix for singlemodal feature (based on eye-tracking) (c) Confusion matrix for multimodal feature (based on the integration of EEG and eye-tracking)

\section{Discussion}

\subsection{Index of mental workload}

The average absolute-frequency band power (delta, theta, alpha, beta and gamma) of all participants can be expressed on a brain topographic map according to the mental workload states induced by two separate emotional designs, as illustrated in Figure 8. This figure can help to identify the corresponding areas of the brain related to variations in mental workload.
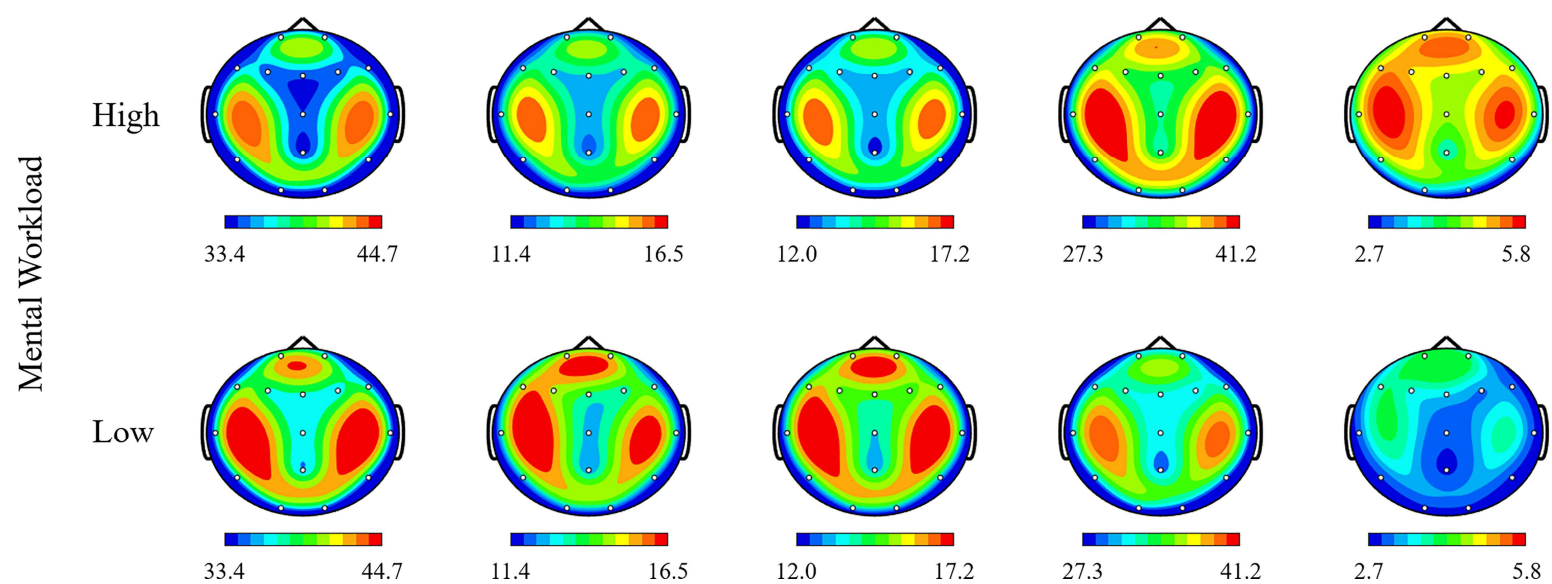

Figure 8: The weight distribution of different brain regions in five frequency bands
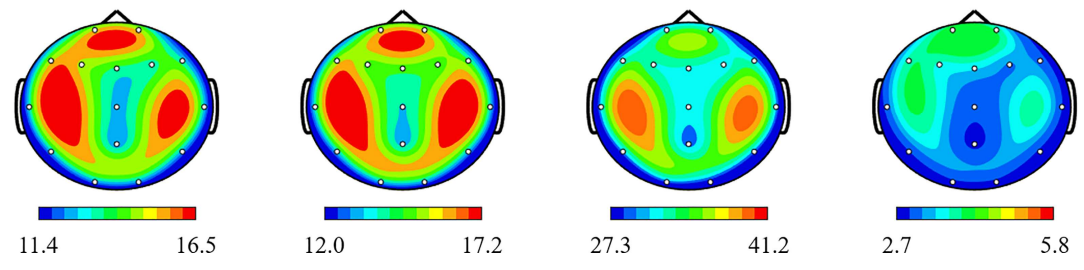

ands 
As shown in Figure 8, whether the mental workload is high or low, delta, theta, alpha and beta all show strong brain activity in the frontal, temporal and occipital lobes. However, gamma displays strong brain activity only in the frontal and temporal lobe. We can speculate that this is due to the fact that the occipital lobe is involved in visual function and the temporal lobe is related to auditory function, which are the main pathways through which participants received information in the E-learning context of the experiment. In addition, the frontal lobes are responsible for focusing attention, and attention plays an important role in E-learning. Moreover, the occipital lobe is involved in feelings, language, memory and abstract thinking, and the anterior temporal lobe is associated with emotions and mental activities.

To validate the classification performance of the single frequency band, we computed the classification accuracies of each frequency band power. Ultimately, we combined five frequency band power features together to the "All" matrix and applied the linear-SVM classifier to this matrix. The corresponding result is presented in Figure 9.

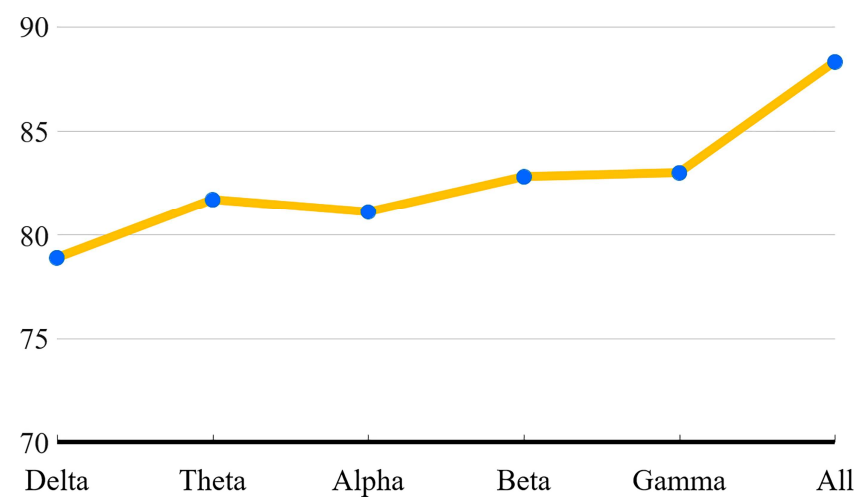

Figure 9: The classification performance of each frequency band and "All" features

Several important observations can be drawn from Figure 9. First, it can be observed that the classification performance of five frequency bands is superior to a single frequency band under the same conditions. Secondly, these results align with past research showing that high frequency bands play a more important role in cognitive activity than low frequency bands[49]. Theta, alpha, beta and gamma all perform well in recognizing and distinguishing high mental workload from low mental workload of the participants, with average classification accuracies all higher than $80 \%$. Compared to previous research, the results of our study reinforce the importance of the gamma frequency band, likely due to the important role emotions play in E-learning[50]. Positive emotions tend to be associated with lower mental workload; moreover, previous studies have shown that the gamma band plays a crucial part in the recognition of emotionswhich appears to be consistent with the above analysis. Therefore, it is reasonable to conclude that emotions are an important measure in evaluating mental workload in E-learning contexts.

According to previous studies conducted by Pope et al.[20], task engagement can be reflected by $\beta /(\theta$ $+\alpha)$. There is a direct correlation between the EEG engagement index and task load [51], the index is very effective in quantifying the state of the mental workload. Based on the above analysis on relevant brain areas and key frequency bands, a similar index for the evaluation of mental workload can be proposed in E-learning contexts, which is rather close to the conclusions drawn by Pope et al.

Mental workload $=\frac{\beta+\gamma}{\theta+\alpha}$

The mental workload curve proposed in this study is illustrated in Figure 10. This figure demonstrates that the index performs well in distinguishing a high mental workload from a low mental workload in the context of E-learning. In addition, the classification performance of the 15-channel index can reach 85.3\%, which greatly reduces the dimensions of the feature while maintaining superior accuracy. 


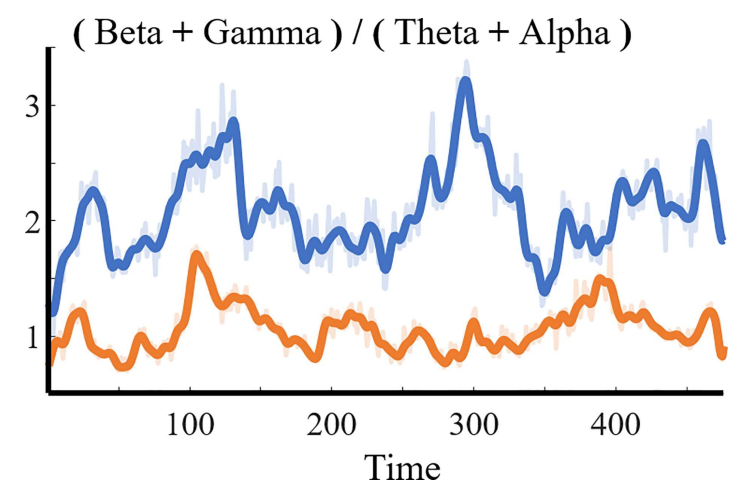

Figure 10: The average (Beta + Gamma) / (Theta + Alpha) of all participants with different emotional designs

\subsection{Result of feature dimensionality reduction}

The main purpose of this research is to identify the relationship between participants' multimodal physiological data (based on EEG and eye-tracking) and different emotional design principles in the context of online courses, and to use machine-learning techniques to classify the mental workload induced by these design differences. This association can be applied to daily life, so it is particularly important that we select the most relevant EEG and eye-tracking features in order to streamline the approach for practical application. The objective of this final phase is to reduce the number of features while maintaining the stability and accuracy of the classification performance.

We calculated the average correlation coefficients between features and labels for all EEG and eyetracking features and sorted them in descending order. Then, a useful matrix was designed, and the features with top-ten correlation coefficients were added to the useful matrix and a linear-SVM was used to work on the useful matrix each time. The corresponding dimensions and classification accuracy percentages are presented in Figure 11.

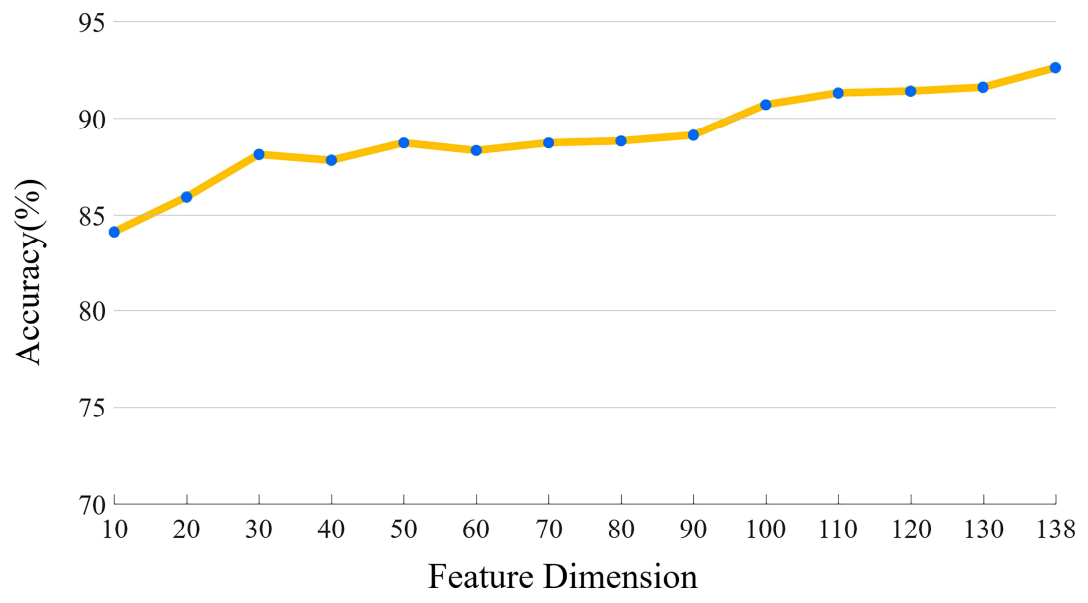

Figure 11: Comparison of feature dimensions and classification performance

As shown in this figure, when the number of dimensions reaches approximately 30 , the classification accuracy becomes notably stable, with an average accuracy of $88.1 \%$. It is evident that the number marking the point of stability is much smaller than the number of original dimensions. Further, the average accuracy of the features ranking in the top 138 , which are independent from the participants, is $92.6 \%$. 


\section{Conclusion}

In this study, EEG and eye-tracking data sets of 42 participants were recorded synchronously while participants engaged with online courses featuring one of two emotional designs hypothesized to have distinct impacts on the learning effect. We investigated multimodal features based on EEG and eye-tracking of these participants, and used machine-learning techniques to identify two states of mental workload aroused by different emotional designs. We extracted three types of features - namely the entropy feature, the wavelet power feature, and the eye-tracking feature - and found that the wavelet power feature demonstrated the best classification performance. Then, we modified the mental workload index proposed by Pope et al. by adding the gamma frequency due to the important role this band plays in the recognition of mental workload in E-learning contexts. The results of the mental workload classification effort using the linear-SVM classifier demonstrate that multimodal physiological features can significantly improve the accuracy of classification, and high frequency bands play a more important role in cognitive activity than low frequency bands in E-learning contexts. In addition, we used correlation to rank all the features in descending order and found that when the feature dimension is reduced to 30 , the optimal average classification accuracy obtained by using the SVM method is $88.1 \%$. The combined recording of EEG and eye-tracking data can provide relevant, objective, and unbiased insights concerning mental load as it changes over time, and can do so without interfering with real-life performance. Continuous monitoring of participants' cognitive state provides not only the promise of instantaneous detection of cognitive overload or underload - but also valuable online feedback to the E-learning system designers and administrators so they may take appropriate countermeasures with the goal of improving the overall learning effect.

Acknowledgement: The author thanks all subjects who participated in this research and the technical support from FISTAR Technology Inc.

Funding Statement: This research was supported by the Research Projects of the Humanities and Social Sciences Foundation of the Ministry of Education of China (20YJA880034), by the Undergraduate Science and Technology Innovation Project of China (202011057019)

Conflicts of Interest: The authors declare that they have no conflicts of interest to report regarding the present study."

\section{References}

[1] Sweller, J. (1999). Instructional design in technical areas. Camberwell. Victoria: ACER Press.

[2] F. Paas, A. Renkl, \& J. Sweller (2004), Cognitive load theory: Instructional implications of the interaction between information structures and cognitive architecture. Instructional science, 32(1/2), 1-8.

[3] Sweller, J. (2010). Element interactivity and intrinsic, extraneous, and germane cognitive load. Educational psychology review, 22(2), 123-138.

[4] Sweller, J., Ayres, P., \& Kalyuga, S. (2011). Intrinsic and extraneous cognitive load. In Cognitive load theory (pp. 57-69). Springer, New York, NY.

[5] Sweller, J., van Merriënboer, J. J., \& Paas, F. (2019). Cognitive architecture and instructional design: 20 years later. Educational Psychology Review, 31(2), 261-292.

[6] Kumar, N., \& Kumar, J. (2016). Measurement of cognitive load in HCI systems using EEG power spectrum: an experimental study. Procedia Computer Science, 84, 70-78.

[7] Holm, A., Lukander, K., Korpela, J., Sallinen, M., \& Müller, K. M. (2009). Estimating brain load from the EEG. The Scientific World JOURNAL, 9, 639-651.

[8] Sauseng, P., Griesmayr, B., Freunberger, R., \& Klimesch, W. (2010). Control mechanisms in working memory: a possible function of EEG theta oscillations. Neuroscience \& Biobehavioral Reviews, 34(7), 1015-1022.

[9] Herweg, N. A., Solomon, E. A., \& Kahana, M. J. (2020). Theta oscillations in human memory. Trends in cognitive sciences, 24(3), 208-227.

[10] Schacter, D. L. (1977). EEG theta waves and psychological phenomena: A review and analysis. Biological psychology, 5(1), 47-82.

[11] Murata, A. (2005). An attempt to evaluate mental workload using wavelet transform of EEG. Human Factors, 47(3), 498-508. 
[12] Brouwer, A. M., Hogervorst, M. A., Holewijn, M., \& van Erp, J. B. (2014). Evidence for effects of task difficulty but not learning on neurophysiological variables associated with effort. International Journal of Psychophysiology, 93(2), 242-252.

[13] Gevins, A., \& Smith, M. E. (2000). Neurophysiological measures of working memory and individual differences in cognitive ability and cognitive style. Cerebral cortex, 10(9), 829-839.

[14] Kahneman, D., \& Beatty, J. (1966). Pupil diameter and load on memory. Science, 154(3756), 1583-1585.

[15] Van Gerven, P. W., Paas, F., Van Merriënboer, J. J., \& Schmidt, H. G. (2004). Memory load and the cognitive pupillary response in aging. Psychophysiology, 41(2), 167-174.

[16] van der Wel, P., \& van Steenbergen, H. (2018). Pupil dilation as an index of effort in cognitive control tasks: A review. Psychonomic bulletin \& review, 25(6), 2005-2015.

[17] Mathôt, S. (2018). Pupillometry: Psychology, physiology, and function. Journal of Cognition, 1(1).

[18] Laeng, B., Sirois, S., \& Gredebäck, G. (2012). Pupillometry: A window to the preconscious?. Perspectives on psychological science, 7(1), 18-27.

[19] Cabestrero, R., Crespo, A., \& Quirós, P. (2009). Pupillary dilation as an index of task demands. Perceptual and motor skills, 109(3), 664-678.

[20] Pope, A. T., Bogart, E. H., \& Bartolome, D. S. (1995). Biocybernetic system evaluates indices of operator engagement in automated task. Biological psychology, 40(1-2), 187-195.

[21] Prinzel, L. J., Freeman, F. G., Scerbo, M. W., Mikulka, P. J., \& Pope, A. T. (2000). A closed-loop system for examining psychophysiological measures for adaptive task allocation. The International journal of aviation psychology, 10(4), 393-410.

[22] Holm, A., Lukander, K., Korpela, J., Sallinen, M., \& Müller, K. M. (2009). Estimating brain load from the EEG. TheScientificWorldJOURNAL, 9, 639-651.

[23] Mayer, R. E., \& Anderson, R. B. (1992). The instructive animation: Helping students build connections between words and pictures in multimedia learning. Journal of educational Psychology, 84(4), 444.

[24] Mayer, R. E., Moreno, R., Boire, M., \& Vagge, S. (1999). Maximizing constructivist learning from multimedia communications by minimizing cognitive load. Journal of educational psychology, 91(4), 638.

[25] Mayer, R. E., \& Chandler, P. (2001). When learning is just a click away: Does simple user interaction foster deeper understanding of multimedia messages?. Journal of educational psychology, 93(2), 390.

[26] Mayer, R., \& Mayer, R. E. (Eds.). (2005). The Cambridge handbook of multimedia learning. Cambridge university press.

[27] Mayer, R. E., \& Pilegard, C. (2005). Principles for managing essential processing in multimedia learning: Segmenting, pretraining, and modality principles. The Cambridge handbook of multimedia learning, 169-182.

[28] Mayer, R. E., \& Fiorella, L. (2014). 12 principles for reducing extraneous processing in multimedia learning: Coherence, signaling, redundancy, spatial contiguity, and temporal contiguity principles. In The Cambridge handbook of multimedia learning (Vol. 279). Cambridge University Press.

[29] Moreno, R. (2007). Optimising learning from animations by minimising cognitive load: Cognitive and affective consequences of signalling and segmentation methods. Applied Cognitive Psychology: The Official Journal of the Society for Applied Research in Memory and Cognition, 21(6), 765-781.

[30] Plass, J. L., Heidig, S., Hayward, E. O., Homer, B. D., \& Um, E. (2014). Emotional design in multimedia learning: Effects of shape and color on affect and learning. Learning and Instruction, 29, 128-140.

[31] Elliot, A. J., \& Maier, M. A. (2012). Color-in-context theory. In Advances in experimental social psychology (Vol. 45, pp. 61-125). Academic Press.

[32] Pekrun, R., Frenzel, A. C., Goetz, T., \& Perry, R. P. (2007). The control-value theory of achievement emotions: An integrative approach to emotions in education. In Emotion in education (pp. 13-36). Academic Press.

[33] Plass, J. L., Heidig, S., Hayward, E. O., Homer, B. D., \& Um, E. (2014). Emotional design in multimedia learning: Effects of shape and color on affect and learning. Learning and Instruction, 29, 128-140.

[34] Um, E., Plass, J. L., Hayward, E. O., \& Homer, B. D. (2012). Emotional design in multimedia learning. Journal of educational psychology, 104(2), 485.

[35] Mayer, R. E., \& Estrella, G. (2014). Benefits of emotional design in multimedia instruction. Learning and Instruction, 33, 12-18.

[36] Hoddes, E., Zarcone, V., Smythe, H., Phillips, R., \& Dement, W. C. (1973). Quantification of sleepiness: a new approach. Psychophysiology, 10(4), 431-436.

[37] Hart, S. G. (2006, October). NASA-task load index (NASA-TLX); 20 years later. In Proceedings of the human factors and ergonomics society annual meeting (Vol. 50, No. 9, pp. 904-908). Sage CA: Los Angeles, CA: Sage publications. 
[38] Hart, S. G., \& Staveland, L. E. (1988). Development of NASA-TLX (Task Load Index): Results of empirical and theoretical research. In Advances in psychology (Vol. 52, pp. 139-183). North-Holland.

[39] Gratton, G., Coles, M. G., \& Donchin, E. (1983). A new method for off-line removal of ocular artifact. Electroencephalography and clinical neurophysiology, 55(4), 468-484.

[40] Jung, T. P., Makeig, S., Humphries, C., Lee, T. W., Mckeown, M. J., Iragui, V., \& Sejnowski, T. J. (2000). Removing electroencephalographic artifacts by blind source separation. Psychophysiology, 37(2), 163-178.

[41] Aftanas, L. I., Lotova, N. V., Koshkarov, V. I., Pokrovskaja, V. L., Popov, S. A., \& Makhnev, V. P. (1997). Nonlinear analysis of emotion EEG: calculation of Kolmogorov entropy and the principal Lyapunov exponent. Neuroscience letters, 226(1), 13-16.

[42] Zhang, C., Wang, H., \& Fu, R. (2013). Automated detection of driver fatigue based on entropy and complexity measures. IEEE Transactions on Intelligent Transportation Systems, 15(1), 168-177.

[43] Pincus, S. M. (1991). Approximate entropy as a measure of system complexity. Proceedings of the National Academy of Sciences, 88(6), 2297-2301.

[44] Pincus, S. (1995). Approximate entropy (ApEn) as a complexity measure. Chaos: An Interdisciplinary Journal of Nonlinear Science, 5(1), 110-117.

[45] Richman, J. S., \& Moorman, J. R. (2000). Physiological time-series analysis using approximate entropy and sample entropy. American Journal of Physiology-Heart and Circulatory Physiology.

[46] Subasi, A. (2007). EEG signal classification using wavelet feature extraction and a mixture of expert model. Expert Systems with Applications, 32(4), 1084-1093.

[47] Motohashi, K., \& Yuan, Y. (2010). Productivity impact of technology spillover from multinationals to local firms: Comparing China's automobile and electronics industries. Research Policy, 39(6), 790-798.

[48] van der Wel, P., \& van Steenbergen, H. (2018). Pupil dilation as an index of effort in cognitive control tasks: A review. Psychonomic bulletin \& review, 25(6), 2005-2015.

[49] Klimesch, W. (1999). EEG alpha and theta oscillations reflect cognitive and memory performance: a review and analysis. Brain research reviews, 29(2-3), 169-195.

[50] Oathes, D. J., Ray, W. J., Yamasaki, A. S., Borkovec, T. D., Castonguay, L. G., Newman, M. G., \& Nitschke, J. (2008). Worry, generalized anxiety disorder, and emotion: Evidence from the EEG gamma band. Biological psychology, 79(2), 165-170.

[51] Berka, C., Levendowski, D. J., Lumicao, M. N., Yau, A., Davis, G., Zivkovic, V. T., ... \& Craven, P. L. (2007). EEG correlates of task engagement and mental workload in vigilance, learning, and memory tasks. Aviation, space, and environmental medicine, 78(5), B231-B244. 